\title{
The life and work of Petrônio Alves Coelho (4 November 1937 - 28 November 2011), with a list of his taxa and publications
}

\author{
Luis Ernesto Arruda Bezerra and Alexandre Oliveira Almeida
}

(LEAB) Universidade Federal de Pernambuco, Departamento de Oceanografia, Programa de Pós-Graduação em Oceanografia. Av. Arquitetura, s/n, Cidade Universitária. 50.670-901 Recife, Pernambuco, Brazil. E-mail: luiseab@gmail.com

(AOA) Universidade Estadual de Santa Cruz, Departamento de Ciências Biológicas. Rod. IlhéusItabuna, km 16, 45662-900 Ilhéus, Bahia, Brazil. E-mail: aalmeida@uesc.br

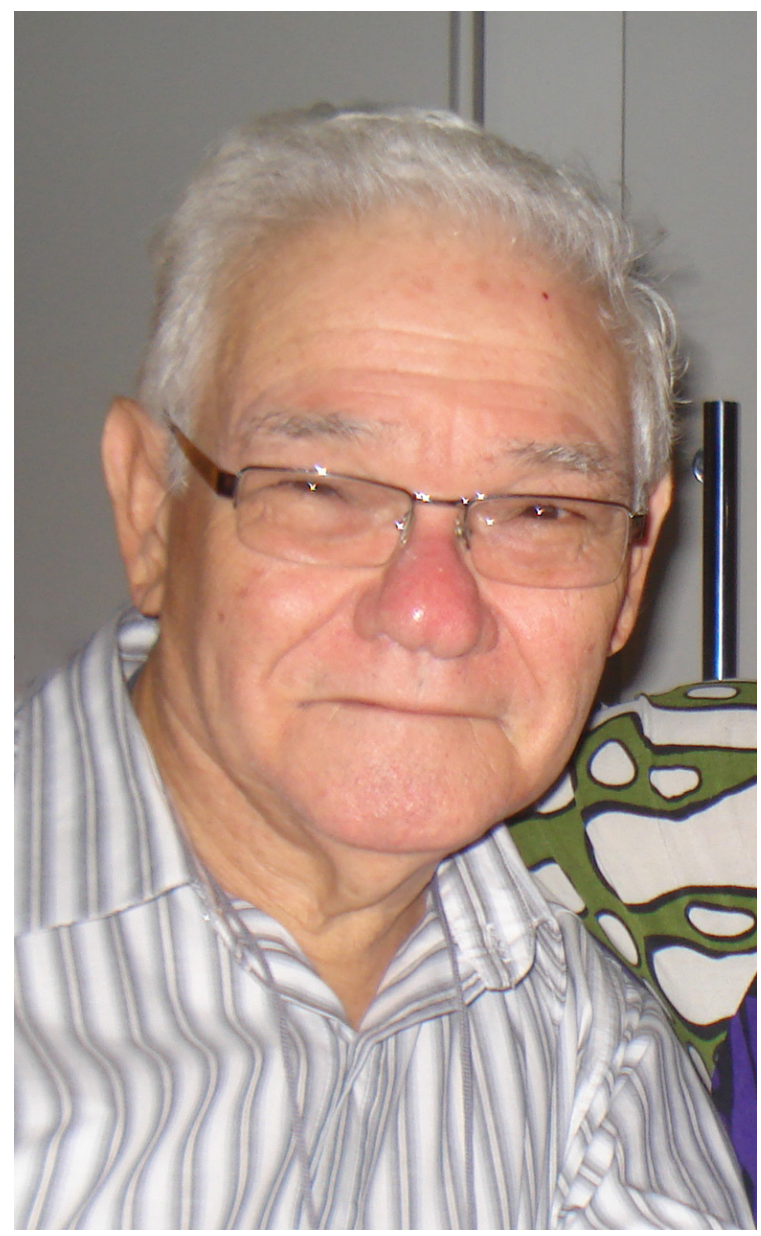

The carcinological community has been greatly saddened to learn that Professor Petrônio Alves Coelho, retired professor at the Departamento de Oceanografia (DOCEAN) of the Universidade Federal de Pernambuco (UFPE), passed away on 28 November 2011, due to a heart attack while recovering from a cerebrovascular accident (CVA). Prof. Petrônio is survived by his wife Maria José
Costa Coelho, four children, two of whom, Petrônio Alves Coelho Filho and Mônica Alves Coelho-Santos, are also carcinologists, and five grandchildren. Prof. Petrônio was born on 4 November 1937 in the city of Recife, the youngest of five children of Heronildes Alves Coelho and Cesina Alves Coelho. He grew up fascinated by a "big ship", which he could see from his house near the Port of Recife. This ship was the Almirante Saldanha, at that time a school ship of the Brazilian Navy, and later a research vessel. Some years later, Prof. Petrônio would take part in several oceanographic expeditions on board this very same ship.

He was graduated in Agronomy from the Universidade Federal Rural de Pernambuco, in 1960. After dedicating several years to the study of insects, especially dragonflies, in 1962 he obtained a position as researcher at the Laboratório de Ciências do Mar (LACIMAR) of UFPE, until 1968, when he became Auxiliary Researcher. Finally, in 1969, he obtained a position as Assistant Professor. In the beginning of his career, he started a crustacean collection that would eventually become the third-largest crustacean collection in Brazil hosted at of UFPE. From the 1960s through the 1980s, Prof. Petrônio took part in important oceanographic expeditions along the Brazilian coast (Appendix 1), including the French Calypso Expedition off the Atlantic coast of South America in 1961 (Forest, 1966). Collections carried out during these expeditions yielded most of the specimens held in the DOCEAN collection. 
From late 1966 through early 1967, he visited several institutions in France and Germany, as part of a technical collaborative program between Brazil and France under the supervision of Dr. Jacques Forest. During his visit to Europe, Prof. Petrônio acquired many papers and references, as well as expertise in decapod taxonomy. After returning to Brazil, he achieved an international reputation and kept in touch with the most important decapod taxonomists of the time, such as Fenner Chace, Jacques Forest, Danièle Guinot, and Lipke Holthuis, among others. His closest colleague and great friend was Dr. Gustavo A. S. de Melo of the Museu de Zoologia, Universidade de São Paulo (USP). The two began a friendship across academic walls, visiting each other frequently and exchanging specimens from their collections. In the early 1970s, he established a long and prolific partnership with Dr. Marilena Ramos-Porto, one of his former students.

In the mid-1970s, the first studies on the viability of freshwater-shrimp farming in Brazil, including testing the farming potential of the indigenous Brazilian species and the introduction of Macrobrachium rosenbergii (De Man, 1879) in 1977, were performed in the Department of Oceanography of UFPE (Cavalcanti, 1998). Before starting these studies, members of the DOCEAN staff visited shrimp farms in Hawaii and Japan to acquire expertise on shrimp farming. Prof. Petrônio actively participated in all these activities. Most of the farming technology first introduced into Brazil was based on practices in Hawaii (Cavalcanti, 1998).

In 1976, Prof. Petrônio obtained his Ph.D. at UFPE, with a thesis on "Biogeography and bionomy of reptantian decapod crustaceans of the Brazilian equatorial region".

In 1982, he was among the founders of the postgraduate course in Oceanography of DOCEAN, which is one of the most important and traditional programs in Brazil. Over the course of his career, he trained and inspired many graduate students in several crustacean groups, including cirripeds, stomatopods, amphipods, tanaids, and decapods; and supervised 35 master's dissertation and eight doctoral thesis projects, as well as uncountable undergraduate students.

Author of over 170 publications, Prof. Petrônio described 29 decapod species and published important papers on the taxonomy, distribution, and biogeography of decapod crustaceans in the South Atlantic, fisheries, and shrimp farming (Appendices 2 and 3). His contributions and enthusiasm helped to make him one of the most important personages in Brazilian zoology, as illustrated by the tributes received from the Brazilian Society of Zoology - SBZ (1998) and the Brazilian Society of Carcinology - SBC (2000 and 2010). Six crustacean species were named in his honor (Appendix 4).

Recently, he was supported by a CNPq scholarship (DCR) to develop research at the Universidade Federal de Alagoas (UFAL) regarding the shrimp fishery in the mouth of the São Francisco River, in Penedo city, together with his son Petrônio Filho.

Prof. Petrônio was an easygoing person, always facing problems with calm and serenity. He was known for his rich sense of humor and for his passion for coffee. He was often seen walking through the department early in the morning, looking for "the poison" as he used to call the coffee, singing and with a smile on his face. Even after he retired, Prof. Petrônio continued to go to the university each day and to advise students interested in crustacean ecology and taxonomy, writing papers and looking for new references in the "crab burrow", as he used to call his office.

Since the beginning of his career, he intended to leave a contribution not only to the science of carcinology, but to Brazilian society, as he wrote:

"In 1960, when I first started the study of crustaceans, very little was known about these species in Brazil. Although the group had been studied by foreign expeditions, the samples obtained would not be as valuable as those collected by local researchers. Having that in mind, I started a research line on ecological distribution and the biology of potential farming species. A few years later, 
other issues arose, related to taxonomy, systematics, and geographic distribution. Additionally, I developed an interest in benthic organisms. The importance of this work relates to several fields of human knowledge. Because they are dominant in aquatic ecosystems, the conservation of crustaceans is very important and therefore their study is essential. Moreover, they are economically important for fisheries, and more recently for aquaculture. Thus, the studies carried out so far as well as the future studies will represent my contribution of productive potential to the well-being of Brazilian society.”

\section{Acknowledgements}

We thank Dr. Fernando Mantelatto for inviting us to write about Prof. Petrônio and suggestions that improved the manuscript, and M.Sc. Débora Lucatelli de Albuquerque and Dr. Jesser Fidelis de Souza-Filho for providing additional information on Prof. Petrônio's life and career. Dr. Janet W. Reind Kindly revised the english text.

\section{References}

Cavalcanti, L.B. 1998. Histórico. p. 17-20. In: W.C. Valenti (ed), Tecnologia para produção de camaróes. Brasília, Instituto Brasileiro do Meio Ambiente e dos Recursos Renováveis.

Forest, J. 1966. Campagne de la Calypso au large des cotes Atlantiques de l'Amérique du Sud (1961-1962) (Première Partie). 1. Compte rendu et list des stations. Annales de l'Institut Océanographique, Monaco, 44: 329-350.

Appendix 1

Oceanographic Expeditions and Main Sampling Programs

1 - Calypso Expedition (1961);

2 - Akaroa (1965);

3 - Canopus (1965/66);

4 - Recife (1965/67);

5 - R/V Almirante Saldanha, Oceanographic
Commission "Norte Nordeste I" (1967);

6 - R/V Almirante Saldanha, Oceanographic Commission "Norte Nordeste II" (1968);

7 - R/V Almirante Saldanha, Oceanographic Commission "Geomar I" (1968);

8 - R/V Almirante Saldanha, Oceanographic Commission "Leste I" (1968);

9 - R/V Almirante Saldanha, Oceanographic Commission "Pesca Norte I" (1968);

10 - Pernambuco Project (1968/69);

11 - R/V Almirante Saldanha, Oceanographic Commission "Sul I" (1969);

12 - Itamaracá Project (1969);

13 - R/V Almirante Saldanha, Oceanographic Commission "Geomar II" (1970);

14 - R/V Almirante Saldanha, Oceanographic Commission "Geomar III" (1971);

15 - B.Pq. Pesquisador IV, Pernambuco Expedition (1971);

16 - R/V Almirante Saldanha, Oceanographic Commission "Sul II" (1972);

17 - FURENE Project (1972);

18 - Paraíba-Pernambuco Project (1969);

19 - B.Pq. Pesquisador IV "Maranhão expedition" (1973);

20 - Condepe/Suape (1978);

21 - R/V Almirante Saldanha, Oceanographic Commission "Nordeste III" (1986);

22 - R/V Almirante Saldanha, Oceanographic Commission "Pavasas I" (1987).

Appendix 2

Publications of Petrônio Alves Coelho

Publications in scientific journals (in chronological order):

Carneiro, O. and Coelho, P.A. 1960. Estudo Ecológico da Barra das Jangadas (Nota Prévia). Trabalhos do Instituto de Biologia Maritima e Oceanografia da Universidade do Recife, 2(1): 237-248.

Coelho, P.A. 1962. Crustáceos decápodos de valor comercial no Estado de Pernambuco. Boletim de Estudos de Pesca, 2(2): 17-18.

Coelho, P.A. 1962. Súmula de observaçôes sobre a lagosta Panulirus argus (Latreille). Boletim de Estudos de Pesca, 2(5): 3-11.

Coelho, P.A. 1962. Sobre a biologia da lagosta cabo-verde. Boletim de Estudos de Pesca, 


\section{2(7): 3-8.}

Coelho, P.A. 1962. Lagostas que ocorrem no Nordeste Brasileiro. Boletim de Estudos de Pesca, 2(7): 13-14.

Coelho, P.A. 1962. Bases para a regulamentação da pesca da lagosta. Boletim de Estudos de pesca, 2(10): 3-6.

Coelho, P.A. 1962. Variaçôes sazonais na composição biológica dos desembarques de lagostas. Boletim de Estudos de Pesca, 3(11/12): 21-32.

Coelho, P.A. and Moura, S.J.C. 1962. Nota sobre as relaçóes existentes entre as medidas de comprimento e peso das lagostas Panulirus argus (Latreille) e P. laevicauda (Latreille) (Crustacea: Decapoda). Trabalhos do Instituto Oceanográfico da Universidade do Recife, 3/4: 53-60. [Dated 1961/1962, published 1962]

Coelho, P.A.; Moura, S.J.C.; Silva, V.R. and Coelho, R.S. 1962. Nota sobre a reprodução das lagostas Panulirus argus (Latreille) e P. laevicauda (Latreille) (Decapoda: Palinuridae) no litoral do Estado de Pernambuco. Trabalhos do Instituto Oceanográfico da Universidade do Recife, 3/4: 61-67. [Dated 1961/1962, published 1962]

Coelho, P.A. 1962. Crescimento da lagosta comum Panulirus argus (Latreille) no litoral do Estado de Pernambuco. Trabalhos do Instituto Oceanográfico da Universidade do Recife, 3/4: 69-73. [Dated 1961/1962, published 1962]

Coelho, P.A. 1962. Observaçóes preliminares sôbre a biologia e a pesca dos camaróes do gênero Macrobrachium Bate, 1868 (Decapoda Palaemonidae) no estado de Pernambuco, Brasil. Trabalhos do Instituto Oceanográfico da Universidade do Recife, 3/4: 75-81. [Dated 1961/1962, published 1962]

Coelho, P.A. 1964. Lista dos Porcellanidae (Crustacea, Decapoda, Anomura) do litoral de Pernambuco e dos estados vizinhos. Trabalhos do Instituto Oceanográfico $d a$ Universidade do Recife, 5/6: 51-68. [Dated 1963/1964, published 1964]
Coelho, P.A. 1964. Algumas observaçôes sôbre a biologia e a ecologia dos camarôes Palaemon northropi e P. pandaliformis no estado de Pernambuco (Decapoda - Palaemonidae). Trabalhos do Instituto Oceanográfico da Universidade do Recife, 5/6: 69-72. [Dated 1963/1964, published 1964]

Coelho, P.A. 1964. Distribuição dos crustáceos decápodos na área de Barra das Jangadas. Trabalhos do Instituto Oceanográfico $d a$ Universidade do Recife, 5/6: 159-173. [Dated 1963/1964, published 1964]

Ottmann, F.; Okuda, T.; Cavalcanti, L.; Silva, O.C.; Araújo, J.V.A.; Coelho, P.A.; Paranaguá, M.N. and Eskinazi, E. 1966. Estudo da Barra das Jangadas - V. Efeitos da poluição sôbre a ecologia do estuário. Trabalhos do Instituto Oceanográfico da Universidade Federal de Pernambuco, 7/8: 7-16. [Dated 1965/1966, published 1966] Coelho, P.A. 1966. Estudo ecológico da lagoa do Ôlho d'Água, Pernambuco, com especial referência aos crustáceos decápodos. Trabalhos do Instituto Oceanográfico da Universidade Federal de Pernambuco, 7/8: 51-70. [Dated 1965/1966, published 1966] Coelho, P.A. 1966. Os crustáceos decápodos de alguns manguezais pernambucanos. Trabalhos do Instituto Oceanográfico da Universidade Federal de Pernambuco, 7/8: 71-90. [Dated 1965/1966, published 1966] Cavalcanti, L.B.; Coelho, P.A.; Kempf, M.; Mabesoone, J.M. and Silva, O.C. 1966. Shelf off Alagoas and Sergipe (Northeastern Brazil). 1. Introduction. Trabalhos do Instituto Oceanográfico da Universidade Federal de Pernambuco, 7/8: 137-150. [Dated 1965/1966, published 1966]

Coelho, P.A. 1969. A distribuição dos crustáceos decápodos reptantes do norte do Brasil. Trabalhos Oceanográficos da Universidade Federal de Pernambuco, 9/11: 223-238. [Dated 1967/1969, published 1969]

Coelho, P.A. 1969. Novas ocorrências de crustáceos decápodos em Pernambuco e estados vizinhos (Brasil). Trabalhos Oceanográficos da Universidade Federal 
de Pernambuco, 9/11: 239-248. [Dated 1967/1969, published 1969]

Coelho, P.A. 1969. Lista dos Stenopodidea (Crustacea Decapoda Natantia) de Pernambuco e estados vizinhos. Trabalhos Oceanográficos da Universidade Federal de Pernambuco, 9/11: 249-254. [Dated 1967/1969, published 1969]

Coelho, P.A. 1971. Nota prévia sôbre os Majidae do norte e nordeste do Brasil (Crustacea, Decapoda). Arquivos do Museu Nacional, 54: 137-145.

Coelho, P.A. 1971. Os crustáceos decápodos reptantes do estuário do Rio Paraíba do Norte. Arquivos do Museu Nacional, 54: 283.

Coelho, P.A. 1972. Descrição de três espécies novas de Majidae do Brasil (Decapoda; Brachyura). Trabalhos Oceanográficos da Universidade Federal de Pernambuco, 13: 119-132.

Coelho, P.A. and Ramos, M.A. 1972. A constituição e a distribuição da fauna de decápodos do litoral leste da América do Sul entre as latitudes de $5^{\circ} \mathrm{N}$ e $39^{\circ} \mathrm{S}$. Trabalhos Oceanográficos da Universidade Federal de Pernambuco, 13: 133-236.

Coelho, P.A. and Koening, M.L. 1972. A distribuição dos crustáceos pertencentes às ordens Stomatopoda, Tanaidacea, Isopoda no norte e nordeste do Brasil. Trabalhos Oceanográficos da Universidade Federal de Pernambuco, 13: 245-260.

Coelho, P.A.; Ramos-Porto, M. and Koening, M.L. 1978. Crustáceos marinhos do Brasil, do Uruguai e da Argentina (ao norte de Mar Del Plata). Consideraçóes biogeográficas. Anais da Universidade Federal Rural de Pernambuco, Recife, 2/3: 227-256. [Dated 1977/1978, published 1978]

Coelho, P.A. and Guedes, D.S. 1979. Estudo ecológico do rio Capibaribe-Mirim. I. Condiçôes gerais da bacia hidrográfica. Trabalhos Oceanográficos da Universidade Federal de Pernambuco, 14: 131-150.

Coelho, P.A.; Macedo, S.J.; Lira, M.E.F. and Guedes, D.S. 1979. Estudo ecológico do rio Capibaribe-Mirim. II. Condiçôes físicas e químicas da água. Trabalhos Oceanográficos da Universidade Federal de Pernambuco, 14: 151-166.

Coelho, P.A. and Ramos-Porto, M. 1980. Bentos litorâneo do Nordeste Oriental do Brasil. I. Povoamentos dos substratos móveis. Boletim do Instituto Oceanográfico, 29(2): 129-131.

Coelho, P.A.; Ramos-Porto, M. 1980. Bentos litorâneo do Nordeste Oriental do Brasil. II. Povoamentos dos substratos duros. Boletim do Instituto Oceanográfico, 29(2): 133-134.

Coelho, P.A. and Ramos-Porto, M. 1980. Crustáceos decápodos da costa do Maranhão, Brasil. Boletim do Instituto Oceanográfico, 29(2): 135-138.

Coelho, P.A. and Santos, M.F.B.A. 1980. Zoogeografia marinha do Brasil. I. Consideraçóes gerais sobre o método e aplicação a um grupo de crustáceos (paguros: Crustacea Decapoda, superfamílias Paguroidea e Coenobitoidea). Boletim do Instituto Oceanográfico, 29(2): 139-144.

Coelho, P.A. and Torres, M.F.A. 1980. Zoogeografia marinha do Brasil. II. Condições ecológicas e biogeográficas sobre a família Leucosiidae (Decapoda, Brachyura). Revista Nordestina de Biologia, 3(especial): 67-77.

Coelho, P.A.; Ramos-Porto, M. and Koening, M.L. 1980. Biogeografia e Bionomia dos crustáceos do litoral equatorial brasileiro. Trabalhos Oceanográficos da Universidade Federal de Pernambuco, 15: 7-138.

Coelho, P.A. and Guedes, D.S. 1980. Estudo ecológico do rio Capibaribe-Mirim: III. Condiçôes biológicas da água. Trabalhos Oceanográficos da Universidade Federal de Pernambuco, 15: 365-378.

Coelho, P.A. 1981. Ocorrência no litoral do Brasil de Scyllarus americanus (Smith) e S. chacei Holthuis (Crustacea, Decapoda, Scyllaridae). Trabalhos Oceanográficos da Universidade Federal de Pernambuco, 16: 13-22.

Coelho, P.A.; Ramos-Porto, M.; Barreto, A.V. and Costa, V.E. 1982. Crescimento em viveiro de cultivo do camarão canela (Macrobrachium amazonicum) (Decapoda, 
Palaemonidae). Revista Brasileira de Zoologia, 1(1): 45-49.

Coelho, P.A. and Torres, M.F.A. 1982. Áreas estuarinas de Pernambuco. Trabalhos Oceanográficos da Universidade Federal de Pernambuco, 17: 67-80.

Coelho, P.A. and Regis, R.A. 1983. Litoral de Alagoas e Sergipe: Crustacea Isopoda. Anais da Sociedade Nordestina de Zoologia, 1(1): 111-116.

Coelho, P.A.; Ramos-Porto, M. and Calado, T.C.S. 1983. Litoral de Alagoas e Sergipe: Decapoda. Anais da Sociedade Nordestina de Zoologia, 1(1): 133-155.

Coelho, P.A. and Torres, M.F.A. 1983. Zoneamento do Estado de Pernambuco para cultivo de camaróes de água doce. Revista Brasileira de Zoologia, 1(3): 149154.

Coelho, P.A. \& Ramos-Porto, M. 1985. Sinopse dos crustáceos decápodos brasileiros (famílias Scyllaridae, Palinuridae, Nephropidae, Parastacidae e Axiidae). Anais da Universidade Federal Rural de Pernambuco, Recife, 8/10: 47-88. [Dated 1983/1985, published 1985]

Coelho, P.A. and Faraj, A.M.P.A. 1985. Ocorrência de Nibilia antilocapra (Stimpson) (Crustacea, Decapoda, Magidae) no nordeste do Brasil. Revista Brasileira de Zoologia, 2(6): 401-404.

Coelho, P.A. and Ramos-Porto, M. 1985. Camaróes de água doce do Brasil: distribuição geográfica. Revista Brasileira de Zoologia, 2(6): 405-410.

Coelho, P.A. and Regis, R.A. 1986. Litoral do Rio Grande do Norte: Crustacea Isopoda. Anais da Sociedade Nordestina de Zoologia, 2(2): 29-37.

Coelho, P.A. and Calado, T.C.S. 1986. Família Albuneidae: distribuição geográfica. Anais da Sociedade Nordestina de Zoologia, 2(2): 39-51.

Coelho, P.A. and Torres, M.F.A. 1986. Crustacea Stomatopoda: Distribuição geográfica no Atlântico Ocidental. Anais $d a$ Sociedade Nordestina de Zoologia, 2(2): 5368.

Coelho, P.A. and Regis, R.A. 1986. Litoral do
Piauí e Ceará: Crustacea Isopoda. Anais da Sociedade Nordestina de Zoologia, 2(2): 6976.

Coelho, P.A. and Ramos-Porto, M. 1986. Sinopse dos crustáceos decápodos brasileiros (famílias Dorippidae e Leucosiidae). Caderno Ômega da Universidade Federal Rural de Pernambuco, Série Ciências Aquáticas, Recife, 2: 67-77.

Coelho, P.A.; Ramos-Porto, M. and Calado, T.C.S. 1986. Litoral do Rio Grande do Norte: Decapoda. Caderno Ômega da Universidade Federal Rural de Pernambuco, Série Ciências Aquáticas, Recife, 2: 79-105. Coelho, P.A. and Ramos-Porto, M. 1986. Sinopse dos crustáceos decápodos brasileiros (famílias Callianassidae, Callianideidae, Upogebiidae, Parapaguridae, Paguridae, Diogenidae). Trabalhos Oceanográficos da Universidade Federal de Pernambuco, 19: 27-53. [Dated 1985/1986, published 1986] Coelho, P.A. 1987. Uma nova espécie de Meticonaxius do Brasil (Crustacea, Decapoda, Callianideidae). Revista Brasileira de Zoologia, 4(1): 63-69.

Coelho, P.A. and Rattacaso, M.C.A. 1988. Revisão das espécies de Upogebia encontradas em Pernambuco, Brasil (Crustacea, Decapoda, Thalassinidea). Revista Brasileira de Zoologia, 5(3): 381392.

Coelho, P.A.; Barreto, A.V. and Costa, K.M.P. 1989. Análise quantitativa de um cultivo de camarão canela Macrobrachium amazonicum (Heller, 1862). Trabalhos Oceanográficos da Universidade Federal de Pernambuco, 20: 203-212. [Dated 1987/1989, published 1989]

Coelho, P.A. and Ramos-Porto, M. 1989. Sinopse dos crustáceos decápodos brasileiros (famílias Dromiidae e Homolidae). Trabalhos Oceanográficos da Universidade Federal de Pernambuco, 20: 213-218. [Dated 1987/1989, published 1989]

Ramos-Porto, M.; Coelho, P.A. and Souza, S.T. 1989. Sinopse dos crustáceos decápodos brasileiros (famílias Penaeidae, Solenoceridae, Sicyoniidae). Trabalhos Oceanográficos da Universidade Federal 
de Pernambuco, 20: 219-234. [Dated 1987/1989, published 1989]

Coelho, P.A. and Regis, R.A. 1990. Litoral do Pará e Maranhão: Crustacea Isopoda. Anais da Sociedade Nordestina de Zoologia, 3: 9-19.

Coelho, P.A.; Ramos-Porto, M. and Melo, G.A.S. 1990. Crustáceos decápodos do estado de Alagoas. Anais da Sociedade Nordestina de Zoologia, 3: 21-34.

Coelho, P.A. and Lacerda, P.R. 1990. Levantamento preliminar dos braquiúros (Crustacea, Decapoda) dos arrecifes da Praia de Piedade, Jaboatão - PE. Anais da Sociedade Nordestina de Zoologia, 3: 35-42.

Coelho, P.A. and Coelho-Santos, M.A. 1990. Crustáceos decápodos e estomatópodos do Rio Paripe, Itamaracá - PE. Anais da Sociedade Nordestina de Zoologia, 3: 43-61.

Coelho, P.A. and Torres, M.F.A. 1990. Revisão das espécies do gênero Mithraculus White na costa atlântica da América do Sul (Crustacea, Decapoda, Majidae). Anais da Sociedade Nordestina de Zoologia, 3: 63-92.

Ramos-Porto, M. and Coelho, P.A. 1990. Sinopse dos crustáceos decápodos brasileiros (família Palaemonidae). Anais da Sociedade Nordestina de Zoologia, 3: 93-111.

Calado, T.C.S.; Coelho, P.A. and RamosPorto, M. 1990. Crustáceos decápodos da superfamília Hippoidea na costa brasileira. Arquivos de Biologia e Tecnologia, 33(4): 743-757.

Coelho, P.A. and Ramos-Porto, M. 1992. Sinopse dos crustáceos decápodos brasileiros (Portunidae). Revista Brasileira de Zoologia, 9(3/4): 291-298.

Coelho, P.A. and Vasconcelos, E.M.S. 1993. Ocorrência de Justitia longimanus (Milne Edwards, 1837), (Crustacea, Decapoda, Palinuridae), no litoral do estado do Rio Grande do Norte - Brasil. Boletim TécnicoCientífico do CEPENE, 1(1): 25-28.

Coelho, P.A. and Coelho Filho, P.A. 1993. Chave para identificação dos crustáceos decápodos braquiúros encontrados nos biótopos de água salobra no litoral oriental do nordeste do Brasil. Boletim TécnicoCientifico do CEPENE, 1(1): 29-56.
Coelho, P.A. and Santos, M.C.F. 1993. Época da reproducáo do camarão rosa, Penaeus subtilis Pérez-Farfante, 1967 (Crustacea, Decapoda, Penaeidae) na regiáo de Tamandaré, PE. Boletim Técnico-Cientifico do CEPENE, 1(1): 57-72.

Coelho, P.A. and Santos, M.C.F. 1993. A pesca de camaróes marinhos ao largo de Tamandaré, PE. Boletim Técnico-Cientifico do CEPENE, 1(1): 73-101.

Coelho, P.A.; Santos, M.C.F. and Botelho, E.R.O. 1993. Alguns peixes ornamentais marinhos ocorrentes no litoral do Município do Rio Formoso - PE. Boletim Técnico-Cientifico do CEPENE, 1(1): 103107.

Coelho, P.A. and Santos, M.C.F. 1993. A pesca de camaróes marinhos no Canal de Santa Cruz - PE. Boletim Técnico-Cientifico do CEPENE, 1(1): 129-155.

Coelho, P.A. and Santos, M.C.F. 1993. Época da reprodução do camaráo branco Penaeus schmitti Burkenroad (Crustacea, Decapoda, Penaeidae) na região de Tamandaré - PE. Boletim Técnico-Cientifico do CEPENE, 1(1): 157-169.

Coelho, P.A. and Santos, M.C.F. 1993. Época de reprodução do camarão setebarbas Xiphopenaeus kroyeri (Heller, 1862) (Crustacea, Decapoda, Penaeidae) na região de Tamandaré - PE. Boletim TécnicoCientifico do CEPENE, 1(1): 171-186.

Coelho, P.A. and Coelho Filho, P.A. 1993. PropostadeclassificaçãodafamíliaXanthidae (Crustacea, Decapoda, Brachyura) através da Taxonomia Numérica. Revista Brasileira de Zoologia, 10(4): 559-580.

Barreto, A.V.; Coelho, P.A. and RamosPorto, M. 1993. Distribuiçáo geográfica dos Brachyura (Crustacea, Decapoda) coletados na plataforma continental do norte e nordeste do Brasil. Revista Brasileira de Zoologia, 10(4): 641-656.

Ramos-Porto, M. and Coelho, P.A. 1993. Sinopse dos crustáceos decápodos brasileiros (família Hippolytidae). Trabalhos Oceanográficos da Universidade Federal de Pernambuco, 22: 181-189. [Dated 1991/1993, published 1993] 
Coelho, P.A. and Torres, M.F.A. 1993. Taxonomia e distribuição das espécies do gênero Acanthonyx Latreille, no Brasil (Crustacea, Decapoda, Majidae). Trabalhos Oceanográficos da Universidade Federal de Pernambuco, 22: 221-241. [Dated 1991/1993, published 1993]

Coelho, P.A. and Coelho-Santos, M.A. 1993. A família Callianassidae no litoral do estado de Pernambuco (Crustacea - Decapoda Thalassinidea). Trabalhos Oceanográficos da Universidade Federal de Pernambuco, 22: 243-257. [Dated 1991/1993, published 1993]

Coelho, P.A. and Coelho Filho, P.A. 1993. Nota sobre a família Carpiliidae no Brasil (Crustacea, Decapoda, Brachyura). Trabalhos Oceanográficos da Universidade Federal de Pernambuco, 22: 259-270. [Dated 1991/1993, published 1993]

Barreto, A.V.; Coelho, P.A. and Melo, G.A.S. 1993. Ocorrência de Acidops cessacii (A. Milne-Edwards, 1878) (Crustacea, Decapoda, Brachyura) no Brasil. Trabalhos Oceanográficos da Universidade Federal de Pernambuco, 22: 271-279. [Dated 1991/1993, published 1993]

Barreto, A.V.; Coelho, P.A. and RamosPorto, M. 1993. Distribuição batimétrica dos Brachyura (Crustacea, Decapoda) na plataforma continental do norte e nordeste do Brasil. Trabalhos Oceanográficos $d a$ Universidade Federal de Pernambuco, 22: 291-303. [Dated 1991/1993, published 1993]

Coelho, P.A. and Coelho-Santos, M.A. 1994. Ocorrência de puerulus de Panulirus Gray (Crustacea, Decapoda, Palinuridae) no nordeste Brasileiro. Boletim TécnicoCientifico do CEPENE, 2(1): 21-24.

Coelho-Santos, M.A. and Coelho, P.A. 1994. Ocorrência dos primeiros estágios juvenis de Panulirus argus (Latreille, 1804) no nordeste Brasileiro (Crustacea, Decapoda, Palinuridae). Boletim Técnico-Cientifico do CEPENE, 2(1): 25-28.

Coelho-Santos, M.A. and Coelho, P.A. 1994. Ocorrência de jovens de Panulirus laevicauda (Latreille, 1817), na Praia de Piedade,
Município de Jaboatão dos Guararapes, Pernambuco, Brasil (Crustacea, Decapoda, Palinuridae). Boletim Técnico-Cientifico do CEPENE, 2(1): 29-33.

Coelho, P.A. and Santos, M.C.F. 1994. Ciclo biológico de Penaeus schmitti Burkenroad, em Pernambuco (Crustacea, Decapoda, Penaeidae). Boletim Técnico-Científico do CEPENE, 2(1): 35-50.

Barreto, A.V. and Coelho, P.A. 1994. Ocorrência de Solenolambrus tenellus Stimpson, 1871 (Crustacea, Decapoda, Parthenopidae) no Brasil. Revista Nordestina de Zoologia, 1(1): 89-100.

Coelho, P.A. and Coelho Filho, P.A. 1994. Taxonomia e distribuição dos Eucratopsinae pertencentes aos gêneros Eurytium, Cyrtoplax e Eurypanopeus (Crustacea, Decapoda, Xanthidae) do litoral brasileiro. Revista Nordestina de Zoologia, 1(1): 101124.

Coelho Filho, P.A.; Coelho-Santos, M.A. and Coelho, P.A. 1994. Estudo dos Xanthidae (Crustacea - Decapoda - Brachyura) da Praia de Piedade, Jaboatão - PE. Revista Nordestina de Zoologia, 1(1): 125-151.

Coelho Filho, P.A. and Coelho, P.A. 1994. Crustacea Decapoda da subfamília Platyxanthinae (Xanthidae) no Litoral do Brasil. Revista Nordestina de Zoologia, 1(1): 152-165.

Coelho-Santos, M.A.; Coelho Filho, P.A. and Coelho, P.A. 1994. Carpiliidae, Eriphiidae e Pilumnidae (Crustacea, Decapoda, Brachyura) da Praia de Piedade, Jaboatão - PE. Revista Nordestina de Zoologia, 1(1): 166-187.

Barreto, A.V. and Coelho, P.A. 1994. Crustacea Decapoda Brachyura coletados durante a Comissão Oceanográfica Pavasas I. Revista Nordestina de Zoologia, 1(1): 188-209.

Coelho, P.A.; Oliveira, J.E.L. and Barbalho, M. 1995. Estágios larvais mais avançados de Panulirus echinatus Smith, 1869 (Decapoda, Palinuridae). Boletim TécnicoCientifico do CEPENE, 3(1): 49-56.

Coelho, P.A. and Ramos-Porto, M. 1995. Crustáceos da regiáo de Tamandaré, estado de Pernambuco, Brasil. Boletim Técnico- 
Cientifico do CEPENE, 3(1): 57-80.

Coelho-Santos, M.A. and Coelho, P.A. 1995. Sazonalidade da ocorrência de crustáceos decápodos no litoral de Jaboatão dos Guararapes, Pernambuco - Brasil. Boletim Técnico-Cientifico do CEPENE, 3(1): 81110.

Coelho, P.A. and Santos, M.A.C. 1995. Resultados das amostragens biológicas na pesca de camaróes marinhos ao largo de Ilhéus, BA. Boletim Técnico-Científico do CEPENE, 3(1): 111-121.

Coelho, P.A. and Santos, M.A.C. 1995. Época da reprodução dos camaróes Penaeus schmitti Burkenroad, 1936 e Penaeus subtilis Pérez-Farfante, 1967 (Crustacea, Decapoda, Penaeidae), na região da foz do rio São Francisco. Boletim Técnico-Cientifico do CEPENE, 3(1): 122-140.

Santos, M.C.F. and Coelho, P.A. 1995. Participação por espécie na pesca artesanal de camarôes marinhos em Tamandaré, PE. Boletim Técnico-Cientifico do CEPENE, 3(1): 141-148.

Coelho, P.A. and Ramos-Porto, M. 1995. Distribuição ecológica dos crustáceos decápodos marinhos do nordeste do Brasil. Trabalhos Oceanográficos da Universidade Federal de Pernambuco, 23: 113-127. [Dated 1994/1995, published 1995]

Ramos-Porto, M. and Coelho, P.A. 1995. Sinopse dos crustáceos decápodos brasileiros: família Pasiphaeidae, gênero Leptochela Stimpson, 1860. Trabalhos Oceanográficos da Universidade Federal de Pernambuco, 23: 129-133. [Dated 1994/1995, published 1995]

Coelho, P.A. 1995. Sinopse dos crustáceos decápodos brasileiros (família Ocypodidae). Trabalhos Oceanográficos da Universidade Federal de Pernambuco, 23: 135-142. [Dated 1994/1995, published 1995]

Coelho Filho, P.A. and Coelho, P.A. 1995. Estudo do gênero Euryozius Miers, 1886 (Crustacea, Decapoda, Brachyura) no Oceano Atlântico. Trabalhos Oceanográficos da Universidade Federal de Pernambuco, 23: 143-148. [Dated 1994/1995, published $1995]$
Coelho, P.A. and Santos, M.C.F. 1995. A pesca de camaróes marinhos ao largo da foz do São Francisco (AL/SE). Trabalhos Oceanográficos da Universidade Federal de Pernambuco, 23: 149-161. [Dated 1994/1995, published 1995]

Coelho-Santos, M.A. and Coelho, P.A. 1995. Diogenidae e Paguridae (Crustacea, Decapoda, Anomura) do litoral de Jaboatáo dos Guararapes, Pernambuco - Brasil. Trabalhos Oceanográficos da Universidade Federal de Pernambuco, 23: 163-176. [Dated 1994/1995, published 1995]

Coelho-Santos, M.A. and Coelho, P.A. 1995. Porcellanidae (Crustacea, Decapoda, Anomura) do litoral de Jaboatáo dos Guararapes, Pernambuco, Brasil. Trabalhos Oceanográficos da Universidade Federal de Pernambuco, 23: 177-191. [Dated 1994/1995, published 1995]

Coelho, P.A.; Dias, A.F.; Oliveira, G.M. and Pontes, A.C.P. 1996. Estudo comparativo da pesca de lagostas com covos, redesde-espera e mergulho no estado de Pernambuco. Boletim Técnico-Cientifico do CEPENE, 4(1): 173-196.

Coelho, P.A. 1996. Distribuição de Mixtopagurus paradoxus A. Milne Edwards, 1880, Pinnixa floridana Rathbun, 1918 e Osachila tuberosa Stimpson, 1871 no litoral brasileiro (Crustacea, Decapoda). Trabalhos Oceanográficos da Universidade Federal de Pernambuco, 24: 165-171.

Coelho, P.A. 1996. Revisão do gênero Alarconia Glassell, 1938, no Brasil, com descrição de Alarconia guinotae, n. sp. (Crustacea, Decapoda, Pinnotheridae). Trabalhos Oceanográficos da Universidade Federal de Pernambuco, 24: 173-178.

Coelho Filho, P.A. and Coelho, P.A. 1996. Sinopse dos crustáceos decápodos brasileiros (família Xanthidae). Trabalhos Oceanográficos da Universidade Federal de Pernambuco, 24: 179-195.

Coelho, P.A. and Santos, M.C.F. 1996. Estudo sobre Xiphopenaeus kroyeri (Heller, 1862) (Crustacea, Decapoda, Penaeidae) em Luís Correia, PI. Trabalhos Oceanográficos da Universidade Federal de Pernambuco, 24: 
241-248.

Coelho, P.A. 1997. Revisão das espécies de Thalassinidea encontradas em Pernambuco, Brasil (Crustacea, Decapoda). Trabalhos Oceanográficos da Universidade Federal de Pernambuco, 25: 137-161.

Coelho, P.A. 1997. Revisão do gênero Pinnixa White, 1846, no Brasil (Crustacea, Decapoda, Pinnotheridae). Trabalhos Oceanográficos da Universidade Federal de Pernambuco, 25: 163-193.

Coelho Filho, P.A. and Coelho, P.A. 1997. Estudo sobre a família Eriphiidae no Brasil (Crustacea - Decapoda - Brachyura). Trabalhos Oceanográficos da Universidade Federal de Pernambuco, 25: 195-203.

Coelho-Santos, M.A. and Coelho, P.A. 1997. Majidae (Crustacea, Decapoda, Brachyura) do litoral de Jaboatão dos Guararapes, Pernambuco - Brasil. Trabalhos Oceanográficos da Universidade Federal de Pernambuco, 25: 205-213.

Coelho-Santos, M.A. and Coelho, P.A. 1997. Portunidae (Crustacea, Decapoda, Brachyura) do litoral de Jaboatão dos Guararapes, Pernambuco - Brasil. Trabalhos Oceanográficos da Universidade Federal de Pernambuco, 25: 215-222.

Coelho, P.A. 1997. Description of Podochela (Anisonotus) atlantica, n. sp., found in the Brazilian and Uruguayan coasts (Crustacea, Decapoda, Majidae). Trabalhos Oceanográficos da Universidade Federal de Pernambuco, 25: 223-226.

Santos, M.C.F. and Coelho, P.A. 1998.

Recrutamento pesqueiro de Xiphopenaeus kroyeri (Heller, 1862) (Crustacea: Decapoda: Penaeidae) na plataforma continental dos estados de Pernambuco, Alagoas e Sergipe - Brasil. Boletim TécnicoCientifico do CEPENE, 6(1): 35-45.

Coelho Filho, P.A. and Coelho, P.A. 1998. Descrição de três espécies novas de Chasmocarcinus Rathbun (Crustacea, Decapoda, Goneplacidae), do litoral brasileiro. Revista Brasileira de Zoologia, 15(3): 799-814.

Coelho-Santos, M.A. and Coelho, P.A. 1998. Camarôes (Crustacea Decapoda) do litoral de Jaboatão dos Guararapes, Pernambuco - Brasil. Trabalhos Oceanográficos da Universidade Federal de Pernambuco, 26(1): 63-83.

Coelho, P.A. 1999. Revisão dos gêneros Eurypodius Guérin, 1825, Anomalothir Miers, 1879 e Eucinetops Stimpson, 1860, nas costas caribe e atlântica da América do Sul (Crustacea, Decapoda, Majidae). Trabalhos Oceanográficos da Universidade Federal de Pernambuco, 27(1): 149-168.

Sousa, E.C.; Coelho, P.A. and Calado, T.C.S. 2000. Crustacea Decapoda dos canais da Lagoa Manguaba no complexo estuarinolagunar Mundaú/Manguaba - Alagoas, Brasil. Boletim de Estudos de Ciências do Mar, 11: 165-192.

Sousa, E.C.; Calado, T.C.S. and Coelho, P.A. 2000. Ocorrência de espécies da família Xanthidae MacLeay, 1838 (Crustacea: Decapoda: Brachyura) no complexo estuarino-lagunar Mundaú/Manguaba - Alagoas, Brasil. Boletim de Estudos de Ciências do Mar, 11: 193-212.

Moura, N.F.O.; Coelho Filho, P.A. and Coelho, P.A. 2000. Population structure of Goniopsis cruentata (Latreille, 1803) in the Paripe estuary, Brazil. Nauplius, 8(1): 7378.

Ramos-Porto, M.; Viana, G.F.S.; Silva, K.C.A.; Cintra, I.H.A. and Coelho, P.A. 2000. Stereomastis sculpta (Smith, 1880) (Decapoda: Polycheloidea: Polychelidae) in Brazilian waters. Nauplius, 8(2): 249-251.

Coelho-Santos, M.A. and Coelho, P.A. 2001. Crustacea Decapoda of the Paripe River Estuary, Pernambuco, Brazil. Hydrobiologia, 449: 77-79.

Coelho, P.A. and Santos, M.C.F. 2002. Contribuição ao conhecimento da lagosta Palinurellus gundlachi von Martens, 1878 no litoral brasileiro (Crustacea, Decapoda, Synaxidae). Boletim Técnico-Cientifico do CEPENE, 10(1): 115-121.

Batista-Leite, L.M.B.; Calado, T.C.S. and Coelho, P.A. 2003. Proporção sexual de três espécies de caranguejos ermitóes (Crustacea, Decapoda, Paguroidea) do Parque Municipal Marinho de Paripueira, 
Alagoas, Brasil. Boletim Técnico-Cientifico do CEPENE, 11(1): 99-108.

Santos, M.C.F. and Coelho, P.A. 2002. Espécies exóticas de camaróes peneídeos (Penaeus monodon Fabricius, 1798 e Litopenaeus vannamei Boone, 1931) nos ambientes estuarino e marinho do nordeste do Brasil. Boletim Técnico-Cientifico do CEPENE, 10(1): 207-222.

Moura, N.F.O.; Coelho, P.A. and Souza, R.F. 2003. A pesca artesanal do aratu, Goniopsis cruentata (Latreille, 1803) (Crustacea, Brachyura, Grapsidae) no litoral norte de Pernambuco - Brasil. Boletim TécnicoCientifico do CEPENE, 11(1): 109-115.

Coelho, P.A. and Santos, M.C.F. 2003. Ocorrência de Charybdis hellerii (Milne Edwards, 1867) (Crustacea, Decapoda, Portunidae) no litoral de Pernambuco. Boletim Técnico-Cientifico do CEPENE, 11(1): 167-173.

Batista-Leite, L.M.A.; Coelho, P.A. and Calado, T.C.S. 2003. Caranguejos-ermitôes (Crustacea, Decapoda, Paguroidea) do Parque Municipal Marinho de Paripueira, Alagoas, Brasil. Boletim Técnico-Cientifico do CEPENE, 11(1): 223-232.

Coelho, P.A. and Lima, I.A. 2003. Cultivo do camarão-pitu, Macrobrachium carcinus (Linnaeus, 1758) (Crustacea, Decapoda, Palaemonidae), em viveiros comerciais. Boletim Técnico-Cientifico do CEPENE, 11(1): 233-244.

Coelho, P.A. and Santos, M.C.F. 2003. Ocorrência de Lysiosquilla glabriuscula (Lamarck, 1818) (Crustacea, Stomatopoda, Lysiosquillidae) no litoral de Pernambuco. Boletim Técnico-Cientifico do CEPENE, 11(1): 293-296.

Barreto, A.V. and Coelho, P.A. 2003. Plataforma continental do estado do Maranhão - Operação Pesquisador IV.II Crustacea, Decapoda, Brachyura. Boletim Técnico-Cientifico do CEPENE, 11(1): 297302.

Vasques, R.O'R.; Almeida, A.O.; Coelho, P.A.; Cuevas, J.M. and Couto, E.C.G. 2003. A previous list of Dendrobranchiata from shrimp trawlings in Ilhéus, Bahia, Brazil.
Nauplius, 11(2): 115-121.

Almeida, A.O.; Coelho, P.A. and Santos, J T.A. 2003. New records of decapod crustaceans (Dendrobranchiata and Brachyura) for the state of Bahia, Brazil. Nauplius, 11(2): 129133.

Moura, N.F.O. and Coelho, P.A. 2003. Fecundidade de Goniopsis cruentata (Latreille, 1803) (Crustacea, Brachyura, Grapsidae) no manguezal do rio Paripe Pernambuco - Brasil. Tropical Oceanography, 31(2): 127-133.

Coelho, P.A.; Santos, M.C.F. and Freitas, A.E.T.S. 2004. Crescimento do aratu-dapedra, Plagusia depressa (Fabricius, 1775) (Crustacea, Decapoda, Plagusiidae), em Tamandaré - Pernambuco. Boletim TécnicoCientífico do CEPENE, 12(1): 73-79.

Coelho, P.A.; Santos, M.C.F.; Freitas, A.E.T.S.; Silva, A.C.C.D. and Santos, S.M. 2004. Crustáceos decápodos coletados no estado de Sergipe - Brasil. Boletim TécnicoCientifico do CEPENE, 12(1): 81-90.

Coelho, P.A. and Santos, M.C.F. 2004. Siris do estuário do rio Una, São José da Coroa Grande, Pernambuco - Brasil (Crustacea, Decapoda, Portunidae). Boletim TécnicoCientifico do CEPENE, 12(1): 187-194.

Moura, N.F.O. and Coelho, P.A. 2004. Maturidade sexual fisiológica em Goniopsis cruentata (Latreille) (Crustacea, Brachyura, Grapsidae) no Estuário do Paripe, Pernambuco, Brasil. Revista Brasileira de Zoologia, 21(4): 1011-1015.

Paiva, A.C.G.; Coelho, P.A. and Torres, M.F.A. 2005. Influência dos fatores abióticos sobre a macrofauna de substratos inconsolidados da zona entre-marés no Canal de Santa Cruz, Pernambuco, Brasil. Arquivos de Ciências do Mar, 38: 85-92.

Coelho, P.A. 2005. Descrição de Austinixa bragantina sp. nov. (Crustacea, Decapoda, Pinnotheridae) do litoral do Pará, Brasil. Revista Brasileira de Zoologia, 22(3): 552555.

Bezerra, L.E.A.; Almeida, A.O. and Coelho, P.A. 2005. Primeiro registro de Apiomithrax violaceus (A. Milne Edwards) e Hypoconcha arcuata Stimpson (Crustacea, Decapoda, 
Brachyura) para o litoral do Ceará. Revista Brasileira de Zoologia, 22(4): 919-922.

Batista-Leite, L.M.A.; Coelho, P.A. and Calado, T.C.S. 2005. Estrutura populacional e utilização de conchas pelo caranguejo ermitão Calcinus tibicen (Herbst, 1791) (Crustacea, Decapoda, Diogenidae). Tropical Oceanography, 33(2): 99-116.

Almeida, A.O.; Coelho, P.A.; Santos, J.T.A. and Ferraz, N.R. 2006. Crustáceos decápodos estuarinos de Ilhéus, Bahia, Brasil. Biota Neotropica, 6(2), http://www. biotaneotropica.org.br/v6n2/pt/abstract?in ventory+bn03406022006.

Santos, M.C.F.; Coelho, P.A. and RamosPorto, M. 2006. Sinopse das informaçóes sobre a biologia e pesca do camarãosete-barbas, Xiphopenaeus kroyeri (Heller, 1862) (Decapoda, Penaeidae) no nordeste do Brasil. Boletim Técnico-Cientifico do CEPENE, 14(1): 141-178.

Lima, J.F.; Abrunhosa, F. and Coelho, P.A. 2006. The larval development of Pinnixa gracilipes Coelho (Decapoda, Pinnotheridae) reared in the laboratory. Revista Brasileira de Zoologia, 23(2): 480489.

Coelho, P.A. 2006. Revisão de Podochela Stimpson e gêneros afins nas costas caribenha e atlântica da América do Sul (Crustacea, Decapoda, Inachidae). Revista Brasileira de Zoologia, 23(3): 678-691.

Bezerra, L.E.A. and Coelho, P.A. 2006. Crustáceos decápodos associados a esponjas no litoral do estado do Ceará, Brasil. Revista Brasileira de Zoologia, 23(3): 699-702.

Bezerra, L.E.A.; Almeida, A.O. and Coelho, P.A. 2006. Occurrence of the family Pinnotheridae De Haan (Crustacea, Decapoda, Brachyura) on the coast of Ceará State, Brazil. Revista Brasileira de Zoologia, 23(4): 1038-1043.

Coelho, P.A.; Almeida, A.O.; Souza-Filho, J.F.; Bezerra, L E.A. and Giraldes, B.W. 2006. Diversity and distribution of the marine and estuarine shrimps (Dendrobranchiata, Stenopodidea and Caridea) from North and Northeast Brazil. Zootaxa, 1221: 4162.
Almeida, A.O.; Coelho, P.A.; Santos, J.T.A. and Ferraz, N.R. 2007. Crustáceos estomatópodos e decápodos da costa de Ilhéus, Bahia, Brasil. Atlântica, 29(1): 5-20.

Santos, M.C.F. and Coelho, P.A. 2007. Crustáceos exóticos reproduzindo em águas costeiras do Nordeste do Brasil. Boletim Técnico-Cientifico do CEPENE, 15(1): 5761.

Almeida, A.O.; Bezerra, L.E.A. and Coelho, P.A. 2007. Further record of Trachypenaeopsis mobilispinis (Rathbun) (Decapoda, Penaeidae) from Brazilian coastal waters. Crustaceana, 80(3): 379-383.

Coelho, P.A.; Almeida, A.O.; Bezerra, L.E.A. and Souza-Filho, J.F. 2007. An updated checklist of decapod crustaceans (infraorders Astacidea, Thalassinidea, Polychelida, Palinura, and Anomura) from the northern and northeastern Brazilian coast. Zootaxa, 1519: 1-16.

Almeida, A.O.; Guerrazi, M.C. and Coelho, P.A. 2007. Stomatopod and decapod crustaceans from Camamu Bay, state of Bahia, Brazil. Zootaxa, 1553: 1-45.

Ramos-Porto, M.; Coelho, P.A.; Silva, K.C.A.; Viana, G.F.S. and Cintra, I.H.A. 2008. Registro da família Eiconaxiidae Sakai \& Ohta, 2005 (Crustacea: Decapoda: Thalassinidea) no Brasil, com redescrição de Eiconaxius carinatus (Bouvier 1925). Biota Neotropica, 8(3): http:// www.biotaneotropica.org.br/v8n3/en/ abstract?Article+ bn00908032008.

Almeida, A.O. and Coelho, P.A. 2008. Estuarine and marine brachyuran crabs (Crustacea: Decapoda) from Bahia, Brazil: checklist and zoogeographical considerations. Latin American Journal of Aquatic Research, 36(2): 183-222.

Almeida, A.O.; Bezerra, L.E.A.; Souza-Filho, J.F.; Almeida, S.M.; Albuquerque, D.L. and Coelho, P.A. 2008. Decapod and stomatopod crustaceans from Santo Aleixo Island, Pernambuco, Brazil. Nauplius, 16(1): 23-41.

Almeida, A.O.; Coelho, P.A.; Luz, J.R.; Santos, J.T.A. and Ferraz, N.R. 2008. Decapod crustaceans in fresh waters of southeastern 
Bahia, Brazil. Revista de Biología Tropical, 56(3): 1225-1254.

Coelho, P.A.; Almeida, A.O. and Bezerra, L.E.A. 2008. Checklist of the marine and estuarine Brachyura (Crustacea: Decapoda) of northern and northeastern Brazil. Zootaxa, 1956: 1-58.

Almeida, A.O.; Santana, F.S.; Paiva, R.J.C.; Souza, G.B.G.; Boehs, G. and Coelho, P.A. 2009. Range extension of the estuarine isopod Cirolana (Anopsilana) jonesi (Crustacea: Cirolanidae) in the western Atlantic Ocean. Marine Biodiversity Records, 2, e150: 1-6.

Larsen, K.; Araújo-Silva, C.L. and Coelho, P.A. 2009. Tanaidacea from Brazil. I. The family Tanaellidae Larsen \& Wilson, 2002. Zootaxa, 2141: 1-19.

Albuquerque, D.L. and Coelho, P.A. 2009. A new species of Nannosquila (Stomatopoda: Nannosquillidae) from the Western Atlantic Ocean. Zootaxa, 2289: 55-60.

Bezerra, L.E.A. and Coelho, P.A. 2010. Redescription of the fiddler crab Uca spinicarpa Rathbun, 1900 (Decapoda: Ocypodidae). Latin American Journal of Aquatic Research, 38(2): 270-273.

Bezerra, L.E.A. and Coelho, P.A. 2010. Intraspecific variation in preserved specimens of the fiddler crabs Uca panacea and Uca pugilator (Decapoda: Ocypodidae). Zoologia, 26(1): 175-182.

Castiglioni, D.S., Oliveira, P.J.A., Silva, J.S. \& Coelho, P.A. 2011. Population dynamics of Sesarma rectum (Crustacea: Brachyura: Grapsidae) in the Ariquindá River mangrove, north-east of Brazil. Journal of the Marine Biological Association of the United Kingdom, 91(7): 1395-1401.

Castiglioni, D.S. \& Coelho, P.A. 2011. Determinaçáo da maturidade sexual de Ucides cordatus (Crustacea, Brachyura, Ucididae) em duas áreas de manguezal do litoral sul de Pernambuco, Brasil. Iheringia, Série Zoologia, 101(1-2): 138-144.

Book chapters:
Coelho, P.A. 1970. Estuários e Lagunas do Nordeste. p. 49-60. In: J. Vasconcelos Sobrinho (org.), As regióes naturais do nordeste, o meio e a civilização. Recife, CONDEPE.

Coelho, P.A. and Ramos-Porto, M. 1982. Crustáceos. p. 329-335. In: Fundação Cidade do Recife (org.), Obras Reunidas C. 1752-1811. Recife, Fundação Cidade do Recife.

Coelho, P.A. and Ramos-Porto, M. 1998. Malacostraca - Eucarida. Stenopodidea. P. 323-324. In: P.S. Young (ed.), Catalogue of Crustacea of Brazil. Rio de Janeiro, Museu Nacional. (Série Livros n. 6)

Ramos-Porto, M. and Coelho, P.A. 1998. Malacostraca - Eucarida. Caridea (Alpheoidea excluded). p. 325-350. In: P.S. Young (ed.), Catalogue of Crustacea of Brazil. Rio de Janeiro, Museu Nacional. (Série Livros n. 6)

Coelho, P.A. and Ramos-Porto, M. 1998. Malacostraca - Eucarida. Palinuridea. p. 387-392. In: P.S. Young (ed.), Catalogue of Crustacea of Brazil. Rio de Janeiro, Museu Nacional. (Série Livros n. 6)

Coelho, P.A.; Paranaguá, M.N.; NeumannLeitão, S.; Melo, R.L.S.; Vasconcelos Filho, A.L. and Oliveira, A.M.E. 1999. Management in northeastern Brazil: faunal diversity. p. 57-67. In: C.A. Brebbia and J.L. Usó (orgs), Ecosystems and sustainable development, Vol. 2. Southampton, WIT Press.

Coelho, P.A. 2000. Carcinofauna. p. 119-127. In: H.M. Barros; E. Eskinazi-Leça; S.J. Macedo and T. Lima (orgs), Gerenciamento Participativo de Estuários e Manguezais. Recife, Quadro Computação Gráfica.

Coelho, P.A.; Coelho-Santos, M.A.; Torres, M.F.A.; Monteiro, B.R. and Almeida, V.A. K. 2002. Reino Animalia: Filo (ou Subfilo) Crustacea no Estado de Pernambuco. p. 429-482. In: M. Tabarelli and J.M.C. Silva (orgs), Diagnóstico da biodiversidade de Pernambuco, Vol. 2. Recife, Secretaria de Ciência, Tecnologia e Meio Ambiente, Editora Massangana.

Coelho, P.A.; Tenório, D.O.; Ramos-Porto, M. 
and Mello, R.L.S. 2004. A fauna bêntica do estado de Pernambuco. p. 477-527. In: E. Eskinazi-Leça; S. Neumann-Leitão and M.F. Costa (orgs), Oceanografia: um cenário tropical. Recife, Ediçóes Bagaço.

Torres, M.F.A.; Ramos-Porto, M. and Coelho, P.A. 2004. Biogeografia marinha. p. 571615. In: E. Eskinazi-Leça; S. NeumannLeitão and M.F. Costa (orgs), Oceanografia: um cenário tropical. Recife, Ediçôes Bagaço.

Coelho, P.A.; Batista-Leite, L.M.A.; CoelhoSantos, M.A. and Torres, M.F.A. 2004. O manguezal. p. 641-688. In: E. EskinaziLeça; S. Neumann-Leitão and M.F. Costa (orgs), Oceanografia: um cenário tropical. Recife, Edições Bagaço.

Coelho, P.A. 2005. Estuários e lagunas do Nordeste. p. 49-60. In: Vasconcelos Sobrinho, J. (org.), As regióes naturais do Nordeste, o meio e a civilização. Recife, Companhia Editora de Pernambuco.

Amaral, A.C.Z.; Ribeiro, C.V.; Santos, S.B.; Avelar, W.; Matthews-Cascon, H.; Leite, F.P.P.; Melo, G.A.S.; Coelho, P.A.; Loureiro, L. and Ventura, R. 2005. Invertebrados aquáticos. p. 99-107. In: A.B.M. Machado; C.S. Martins and G.M. Drummond (orgs), Lista da fauna brasileira ameaçada de extinção. Belo Horizonte, Fundação Biodiversitas.

Amaral, A.C.Z.; Ribeiro, C.V.; Mansur, M.C.D.; Santos, S.B.; Avelar, W.E.P.; Matthews-Cascon, H.; Leite, F.P.P.; Melo, G.A.S.; Coelho, P.A.; Bond-Buckup, G.; Bubkup, L.; Ventura, C.R.R. and Tiago, C.G. 2008. A Situação de Ameaça dos Invertebrados Aquáticos no Brasil. p. 157-165. In: A.B.M. Machado; G.M. Drummond and A.P. Paglia (orgs), Livro vermelho da fauna brasileira ameaçada de extinção, Vol. 1. Brasília, Ministério do Meio Ambiente; Belo Horizonte, Fundação Biodiversitas.

Coelho, P.A. and Melo, G.A.S. 2008. Atya gabonensis Giebel, 1875. p. 270-271. In: A.B.M. Machado; G.M. Drummond and A.P. Paglia (orgs), Livro vermelho da fauna brasileira ameaçada de extinção, Vol. 1. Brasília, Ministério do Meio Ambiente;
Belo Horizonte, Fundação Biodiversitas. Melo, G.A.S. and Coelho, P.A. 2008. Atya scabra (Leach, 1815). p. 272-273. In: A.B.M. Machado; G.M. Drummond and A.P. Paglia (orgs), Livro vermelho da fauna brasileira ameaçada de extinção, Vol. 1. Brasília, Ministério do Meio Ambiente; Belo Horizonte, Fundação Biodiversitas.

Coelho, P.A. and Melo, G.A.S. 2008. Gecarcinus lagostoma H. Milne Edwards, 1835. p. 274-275. In: A.B.M. Machado; G.M. Drummond and A.P. Paglia (orgs), Livro vermelho da fauna brasileira ameaçada de extinção, Vol. 1. Brasília, Ministério do Meio Ambiente; Belo Horizonte, Fundação Biodiversitas.

Coelho, P.A. and Melo, G.A.S. 2008. Percnon gibbesii (H. Milne Edwards, 1853). p. 275-276. In: A.B.M. Machado; G.M. Drummond and A.P. Paglia (orgs), Livro vermelho da fauna brasileira ameaçada de extinção, Vol. 1. Brasília, Ministério do Meio Ambiente; Belo Horizonte, Fundação Biodiversitas.

Melo, G.A.S. and Coelho, P.A. 2008. Macrobrachium carcinus (Linnaeus, 1758). p. 277-278. In: A.B.M. Machado; G.M. Drummond and A.P. Paglia (orgs), Livro vermelho da fauna brasileira ameaçada de extinção, Vol. 1. Brasília, Ministério do Meio Ambiente; Belo Horizonte, Fundação Biodiversitas.

Melo, G.A.S. and Coelho, P.A. 2008. Minyocerus angustus (Dana, 1852). p. 279-280. In: A.B.M. Machado; G.M. Drummond and A.P. Paglia (orgs), Livro vermelho da fauna brasileira ameaçada de extinção, Vol. 1. Brasília, Ministério do Meio Ambiente; Belo Horizonte, Fundação Biodiversitas.

Published and organized/edited books:

Coelho, P.A.; Ramos-Porto, M. and Soares, C.M.A. 1981. Cultivo de camaróes do gênero Macrobrachium Bate (Decapoda, Palaemonidae) no Brasil. Natal, Empresa de Pesquisa Agropecuária do Rio Grande do Norte, 66p. 
Coelho, P.A.; Ramos-Porto, M. and Soares, C.M.A. 1982. Biologia e Cultivo de Camarôes de Água Doce. Recife, Universidade Federal de Pernambuco, 53p.

Macedo, S.J.; Cavalcanti, L.B.; EskinaziLeça, E.; Paranaguá, M.N.; Coelho, P.A. and Luna, J.A.C. 1983. Caracterização do Complexo Estuarino-Lagunar da Área de Suape (Pernambuco-Brasil). Volume 1. Síntese Ecológica. Recife, CONDEPE, $107 \mathrm{p}$.

Coelho, P.A. and Coelho-Santos, M.A. 1999. A estória do pássaro Viajante que descobriu os crustáceos do Canal de Santa Cruz. Recife, Universidade Federal Rural de Pernambuco, 16p.

Coelho, P.A. 2000. Boletim de estatística da produção de pescado no Baixo São Francisco. Maceió - AL. v. 1. 21p.

Species descriptions published in congresses communications books:

Coelho, P.A. 1972. Descrição preliminar de uma espécie nova de $U c a$ de Pernambuco e Paraíba. In: V Congresso Brasileiro de Zoologia, 1972, São Paulo. Resumos, 1972. p. 42.

Coelho, P.A. 1973. Descrição preliminar de Ebalia conica n. sp., do Norte e Nordeste do Brasil (Crustacea, Decapoda, Leucosiidae). Ciência e Cultura, 25(6)(Supl.): 342.

Coelho, P.A. 1973. Descrição preliminar de Ebalia obliqua, n. sp., do Norte e Nordeste do Brasil (Crustacea, Decapoda, Leucosiidae). Ciência e Cultura, 25(6) (Supl.): 342-343.

Coelho, P.A. 1973. Descrição preliminar de Ebalia vertiginosa, n. sp., do Norte e Nordeste do Brasil (Crustacea, Decapoda, Leucosiidae). Ciência e Cultura, 25(6) (Supl.): 343.

Coelho, P.A. 1973. Descrição preliminar de Clythrocerus carinatus, n. sp., do Norte e Nordeste do Brasil (Crustacea, Decapoda, Dorippidae). Ciência e Cultura, 25(6) (Supl.): 343.

Coelho, P.A. 1973. Descrição preliminar de Clythrocerus analogus, n. sp., do litoral brasileiro (Crustacea, Decapoda, Dorippidae). Ciência e Cultura, 25(6) (Supl.): 343-344.

Coelho, P.A. 1973. Descrição preliminar de Munida brasiliae, n. sp., do Norte e Nordeste do Brasil (Crustacea, Decapoda, Galatheidae). Ciência e Cultura, 25(6) (Supl.): 344.

Coelho, P.A. 1973. Descrição preliminar de Cupogebia (sic) (Calliadne) acanthura, n. sp., do Brasil (Crustacea, Decapoda, Callianassidae). Ciência e Cultura, 25(6) (Supl.): 344.

Coelho, P.A. 1973. Descrição preliminar de Calastacus angulatus, n. sp., e de $C$. spinosus, n. sp., do Norte do Brasil (Crust., Decapoda, Axiidae). Ciência e Cultura, 25(6)(Supl.): 344-345.

Coelho, P.A. 1973. Descrição preliminar de Upogebia (Upogebia) marina, n. sp., do Nordeste do Brasil (Crustacea, Decapoda, Callianassidae). Ciência e Cultura, 25(6) (Supl.): 345.

Coelho, P.A. 1973. Descrição preliminar de Meticonaxius minutus, n. sp., do Norte do Brasil (Crustacea, Decapoda, Axiidae). Ciência e Cultura, 25(6)(Supl.): 345.

Appendix III

Taxa erected by or with Petrônio Alves Coelho

Decapoda - Stomatopoda

Nannosquilla potiguara Albuquerque \& Coelho, 2009

Decapoda - Axiidea

Acanthaxius angulata (Coelho, 1973)* (as Calastacus angulatus)

Axiopsis brasiliensis Coelho \& Ramos-Porto, 1985 [as Axiopsis (Axiopsis) brasiliensis]

Calaxius spinosus (Coelho, 1973)* (as Calastacus spinosus)

Marcusiaxius minutus (Coelho, 1973) (as Meticonaxius minutus)

Meticonaxius capricorni Coelho, 1987

Paraxiopsis vicina (Coelho \& Ramos-Porto, 1985) [as Axiopsis (Paraxiopsis) vicina] 
* Genus assignment following Coelho et al. (2007) [Coelho, P.A.; Almeida, A.O.; Bezerra, L.E.A. and Souza-Filho, J.F. 2007. An updated checklist of decapod crustaceans (infraorders Astacidea, Thalassinidea, Polychelidae, Palinura and Anomura) from the northern and northeastern Brazilian coast. Zootaxa, 1519: 1-16].

Decapoda - Gebiidea

Upogebia acanthura Coelho, 1973 [as Upogebia (Calliadne) acanthura]

Upogebia marina Coelho, 1973 [as Upogebia (Upogebia) marina]

Decapoda - Anomura

Munida brasiliae Coelho, 1973 (junior synonym of Munida angulata Benedict, 1902)

Decapoda - Brachyura

Acanthonyx dissimulatus Coelho, 1993

Alarconia guinotae Coelho, 1996

Anisonotus atlanticus (Coelho, 1997) (as Podochela atlantica)

Austinixa bragantina Coelho, 2005

Austinixa leptodactyla (Coelho, 1997) (as Pinnixa leptodactyla)

Chasmocarcinus arcuatus Coelho Filho \& Coelho, 1998

Chasmocarcinus hirsutipes Coelho Filho \& Coelho, 1998

Chasmocarcinus meloi Coelho Filho \& Coelho, 1998

Clythrocerus carinatus Coelho, 1973

Deilocerus analogus (Coelho, 1973) (as Clythrocerus analogus)

Epialtoides rostratus Coelho, 1972

Ericerodes minusculus (Coelho, 1972) (as Podochela minuscula)

Lithadia conica (Coelho, 1973) (as Ebalia conica)

Lithadia obliqua (Coelho, 1973) (as Ebalia obliqua)

Lithadia vertiginosa (Coelho, 1973) (as Ebalia vertiginosa)

Pinnixa gracilipes Coelho, 1997

Pinnixa latissima Coelho, 1997

Podochela brasiliensis Coelho, 1972
Uca panema Coelho, 1972 [junior synonym of Uca (Minuca) burgersi Holthuis, 1967]

Appendix III

Species named for Petrônio Alves Coelho:

Tanaidacea

Acutihumerus petronius Araújo-Silva \& Larsen, 2010

Decapoda - Caridea

Leptalpheus petronii Ramos-Porto \& Souza, 1994 (nomen dubium*)

Macrobrachium petronioi Melo, Lobáo \& Fernandes, 1996

Decapoda - Anomura

Munida petronioi Melo-Filho \& Melo, 1994

Decapoda - Brachyura

Deilocerus coelhoi Campos Jr. \& Melo, 1998

Pilumnoides coelhoi Guinot \& Macpherson, 1987

* According to De Grave \& Fransen (2011) [De Grave, S. and Fransen, C.H.J.M. 2011. Carideorum catalogus: the recent species of the dendrobranchiate, stenopodidean, procarididean and caridean shrimps (Crustacea: Decapoda). Zoologische Mededelingen, 85(9): 195-589]. 